\title{
Application of optical beams to electrons in graphene
}

\author{
A. Matulis,,$^{1,2, *}$ M. Ramezani Masir,,${ }^{1 \dagger}$ and F. M. Peeters ${ }^{1, \ddagger}$ \\ ${ }^{1}$ Departement Fysica, Universiteit Antwerpen, Groenenborgerlaan 171, B-2020 Antwerpen, Belgium \\ ${ }^{2}$ Semiconductor Physics Institute, Center of Physical Sciences and Technology, Goštauto 11, LT-01108 Vilnius, Lithuania
}

(Received 3 November 2010; revised manuscript received 19 January 2011; published 29 March 2011)

\begin{abstract}
The technique of beam optics is applied to the description of the wave function of Dirac electrons. This approach is illustrated by considering electron transmission through simple nonhomogeneous structures, such as flat and bent $p$ - $n$ junctions and superlattices. We found that a convex $p$ - $n$ junction compresses the beam waist, while a concave interface widens it without loosing its focusing properties. At a flat $p$ - $n$ junction the waist of the transmitted Gaussian beam can be narrowed or widened, depending on the angle of incidence. A general condition is derived for the occurrence of beam collimation in a superlattice which is less stringent than previous discussed.
\end{abstract}

DOI: 10.1103/PhysRevB.83.115458

PACS number(s): 81.05.ue, 72.80.Vp, 41.75.-i, 73.21.Cd

\section{INTRODUCTION}

Progress in nanometer technology has triggered a broad activity in low-dimensional quantum systems. ${ }^{1}$ Starting with two-dimensional (2D) electron systems at the interface of two materials several decades ago, it has shifted recently to 2D materials, for example, graphene, ${ }^{2,3}$ and 2D topological insulators. ${ }^{4}$ The most intriguing feature of these systems is their similarity to ultrarelativistic electrons and positrons obeying the Dirac equation. ${ }^{5}$

The possibility of performing experiments at very low temperatures with pure materials stimulated investigations of sophisticated quantum properties of electron systems, especially the peculiarities of the electron wave functions, their control, and the search for analogies with electromagnetic waves. For instance, the meta-material character of $p-n$ structures in graphene ${ }^{6}$ was pointed out, and focusing of electronic waves was proposed. ${ }^{7,8}$ The meta-material properties of the above-mentioned $p-n$ structures resulted in the expectancy of controlling the electron wave function, in particular, the width of electron beams by means of a superlattice. This behavior is known as collimation. Recently it was shown that superlattices on graphene can be fabricated in various ways ${ }^{9-12}$ which induce large theoretical activity in this field, such as the investigation of electron focusing, ${ }^{13-15}$ collimation of electrons and photons, ${ }^{16-21}$ and interference ${ }^{22}$ in a $2 \mathrm{D}$ electron gas.

Qualitatively, the meta-material properties of $p-n$ junctions in graphene can be understood by inspecting classical trajectories or using ray optics, as it is called for the case of electromagnetic phenomena. A quantitative description of their properties can be achieved by laborious numerical simulation $^{23,24}$ of the Schrödinger and Dirac equations (similar to the Maxwell equations for describing electromagnetic phenomena).

In photonics an intermediate approach based on beam optics $^{25}$ has been widely used, in particular, in laser physics. Beam optics is based on the simplicity of ray optics, but with the inclusion of essential phenomena such as diffraction and interference. Compared to mechanics it corresponds to the so-called quasiclassical approach.

The aim of this work is to transfer the technique of Gaussian beams developed in optics to the description of Dirac electron wave functions and to illustrate their application in electron propagation through the most simple nonhomogeneous structures, such as flat and bent $p$ - $n$ junctions and superlattices. The main advantage of the present approach compared with the previous calculations is its simplicity and the fact that most of the results can be obtained analytically.

The paper is organized as follows. In Sec. II the main properties of Gaussian beams in optics are reviewed. Gaussian beams for Dirac electrons are introduced in Sec. III. Their application to beam focusing by a bent $p$ - $n$ junction is discussed in Sec. IV. Section V is devoted to the reflection of oblique beams, and in Sec. VI beam collimation by a superlattice is discussed. In the last section our conclusions are presented.

\section{GAUSSIAN BEAMS IN OPTICS}

We first review the basic properties of Gaussian beams in optics, which are essential when solving the Dirac equation for the corresponding electron beams in graphene. Following Saleh's textbook, ${ }^{25}$ Gaussian beams are known as solutions of the three-dimensional (3D) Helmholtz equation,

$$
\left(\nabla^{2}+k^{2}\right) u(\mathbf{r})=0,
$$

in the form of paraxial waves

$$
u(\mathbf{r})=A(\mathbf{r}) \mathrm{e}^{i k x}
$$

propagating along the $x$ axis. The envelope $A(\mathbf{r})$ is assumed to be a slowly varying function over distances of the order of the wavelength $\lambda=2 \pi / k$. This envelope satisfies the paraxial Helmholtz equation

$$
\left(\nabla_{\perp}^{2}+2 i k \frac{\partial}{\partial x}\right) A(\mathbf{r})=0,
$$

where $\nabla_{\perp}^{2}=\partial^{2} / \partial y^{2}+\partial^{2} / \partial z^{2}$ stands for the 2D Laplacian in the plane perpendicular to the direction of beam propagation. Inspecting terms in the above equation and comparing them with the terms in Eq. (1), we conclude that the validity of the paraxial approximation can be formally expressed as

$$
k^{-1} \partial_{x} A=\gamma A \ll A, \quad \partial_{\perp}^{2} A \sim k \partial_{x} A \sim \gamma k^{2} A .
$$

So in the paraxial approximation, terms of order $\gamma$ are taken into account while terms of order $\gamma^{2}$ are omitted, or only the leading terms are taken into account. 


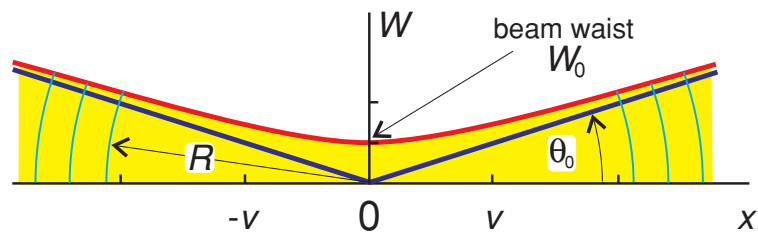

FIG. 1. (Color online) Illustration of a Gaussian beam with its important parameters.

Making use of the analogy with the Green's function of the diffusion equation for imaginary time (e.g., $t \rightarrow i x / 2 k$ ), the solution of Eq. (3) can be presented as

$$
A(\mathbf{r})=\frac{A}{x-w} \exp \left\{\frac{i k \rho^{2}}{2(x-w)}\right\},
$$

where $\rho=\sqrt{y^{2}+z^{2}}$ is the radial coordinate in the $y z$ plane, and

$$
w=u+i v
$$

is some complex shift of the argument, which can be included due to the translational symmetry of Eq. (3). The above complex constant allows us to obtain a finite beam solution in the $y z$ plane.

Usually the denominator in the argument of the exponent is decomposed as follows:

$$
\begin{aligned}
\frac{1}{x-w} & =\frac{1}{x-u-i v}=\frac{x-u}{(x-u)^{2}+v^{2}}+\frac{i v}{(x-u)^{2}+v^{2}} \\
& =\frac{1}{R(x-u)}+\frac{2 i}{k W^{2}(x-u)},
\end{aligned}
$$

where the symbol $W(x)$ stands for the beam radius and $R(x)$ for the wave-front radius of curvature. The smallest beam radius,

$$
W_{0}=W(0)=\sqrt{\frac{2 v}{k}},
$$

is called the beam waist, while the parameter $2 v$ characterizes the depth of focus and is called the confocal parameter. The parameter $u$ is just the position of the waist on the $x$ axis. All these quantities are shown in Fig. 1. The beam radius $W(x)$ is shown by the red curve. In the limiting case of $x \rightarrow \pm \infty$ it approaches the two blue lines indicating the angular divergence of the beam:

$$
\theta_{0}=\frac{2}{k W_{0}} .
$$

Taking into account condition (4) it is easy to conclude that the paraxial approximation is valid when

$$
\frac{\partial A}{\partial x} \ll k A \quad \text { or } \quad \theta_{0} \ll 1,
$$

namely, in the case of weakly diverging beams.

The intensity of the beam can be calculated in a standard way as

$$
\begin{aligned}
I(\rho, x) & =|u(\mathbf{r})|^{2}=\frac{I_{0}}{(x-u)^{2}+v^{2}} \exp \left[-\frac{k \rho^{2} v}{(x-u)^{2}+v^{2}}\right] \\
& =I_{0}\left[\frac{W_{0}}{W^{2}(x-u)}\right]^{2} \exp \left[-\frac{2 \rho^{2}}{W^{2}(x-u)}\right]
\end{aligned}
$$

where $I_{0}=|A|^{2} / v^{2}$ characterizes the total optical power $\left(\pi W_{0}^{2} I_{0} / 2\right)$ of the beam. Equation (11) actually justifies the name of the beam's radius $W(x)$.

All these expressions can be straightforwardly applied to $3 \mathrm{D}$ electron beams that are described by the Schrödinger equation. For free electrons this equation coincides with the Helmholtz equation (1) if one takes into account the definition of the electron energy, namely, $E=\hbar^{2} k^{2} / 2$.

\section{GAUSSIAN BEAMS APPLIED TO THE DIRAC EQUATION}

Now we turn to the Dirac-Weyl equation, which is used in a continuum description of electrons and holes in graphene or for electrons in topological insulators. In the stationary case this equation can be presented in the form of a Schrödinger equation,

$$
(H-E) \Psi(\mathbf{r})=0,
$$

with the following dimensionless 2D Hamiltonian:

$$
H=-i\left(\begin{array}{cc}
0 & \partial / \partial x-i \partial / \partial y \\
\partial / \partial x+i \partial / \partial y & 0
\end{array}\right) .
$$

All dimensions can be excluded, say, measuring distances in lattice constant $a_{0}$ units, time in $\hbar / a_{0}$ units, and energy in $\hbar v_{F} / a_{0}$ units (here $v_{F}$ is the Fermi velocity-the single parameter characterizing the Dirac Hamiltonian for particles with zero rest mass).

Equation (12) has to be satisfied by the two-component eigenfunction,

$$
\Psi(\mathbf{r})=\Psi(x, y)=\left(\begin{array}{l}
F(\mathbf{r}) \\
G(\mathbf{r})
\end{array}\right),
$$

where the components obey the following set of equations:

$$
\begin{aligned}
& \left(\frac{\partial}{\partial x}-i \frac{\partial}{\partial y}\right) G(\mathbf{r})=i E F(\mathbf{r}), \\
& \left(\frac{\partial}{\partial x}+i \frac{\partial}{\partial y}\right) F(\mathbf{r})=i E G(\mathbf{r}) .
\end{aligned}
$$

Substituting the component $G(\mathbf{r})$ (expressed from the second equation) into the first one, we arrive at the equation for the single $F(\mathbf{r})$ component,

$$
\left(\frac{\partial^{2}}{\partial x^{2}}+\frac{\partial^{2}}{\partial y^{2}}+E^{2}\right) F(\mathbf{r})=0,
$$

which coincides with the 2D Helmholtz equation, (1), or the Schrödinger equation for standard electrons, if we take into account the electron and hole energy dependence

$$
E= \pm k
$$

in graphene.

The different dimensionality of the problem changes the paraxial equation slightly for the envelope functions introduced as

$$
F(\mathbf{r})=e^{i k x} f(\mathbf{r}), \quad G(\mathbf{r})=e^{i k x} g(\mathbf{r})
$$


for the beam propagating along the $x$ axis. Now instead of Eq. (3) we have to write down the following equation for the first envelope function component:

$$
\left(\frac{\partial^{2}}{\partial y^{2}}+2 i k \frac{\partial}{\partial x}\right) f(\mathbf{r})=0 .
$$

The solution of Eq. (19), in analogy with the Gaussian beam in optics, Eq. (5), can be presented as

$$
f(\mathbf{r})=\frac{A}{\sqrt{x-w}} \exp \left\{\frac{i k y^{2}}{2(x-w)}\right\} .
$$

We see that the exponents that define the main properties of the Gaussian beam are the same in both Eq. (20) and Eq. (5). Only the prefactors are different, which is caused by the peculiarities of the Green's function of the 1D diffusion equation.

The second envelope function component can be obtained using Eqs. (15b) and (18). It reads

$$
\begin{aligned}
g & =-\frac{i}{E} \mathrm{e}^{-i k x}\left(\frac{\partial}{\partial x}+i \frac{\partial}{\partial y}\right) \mathrm{e}^{i k x} f \\
& =-\frac{i}{E}\left(i k+\partial_{x}+i \partial_{y}\right) f \approx \frac{k}{E} f .
\end{aligned}
$$

We restrict our consideration by taking into account only the last approximate expression or including just the leading term, which, according to Eq. (4), is the essence of the paraxial approximation. Thus, the Gaussian beam propagating along the $x$ axis, being the approximate solution of the Dirac equation, can be presented as

$$
\Psi(\mathbf{r})=\frac{A e^{i k x}}{\sqrt{x-w}}\left(\begin{array}{l}
k \\
E
\end{array}\right) \exp \left\{\frac{i k y^{2}}{2(x-w)}\right\} .
$$

If the beam propagates in some other direction, its wave function can be obtained from Eq. (22) by properly rotating the $(x, y)$ coordinates and the spinor part of the wave function as well.

\section{TRANSMISSION OF AN ELECTRON BEAM THROUGH A BENT POTENTIAL STEP}

For illustrative purposes we use the expressions obtained in the previous section for Dirac electron Gaussian beams and apply them to the penetration of these electrons into a bent electric potential step in graphene. We assume that the $x y$ plane is divided into two parts by the circular interface

$$
\left(x-R_{0}\right)^{2}+y^{2}=R_{0}^{2} .
$$

In the vicinity of a narrow Gaussian beam propagating along the $x$ axis, it can be replaced by the parabolic curve

$$
x=\frac{y^{2}}{2 R_{0}},
$$

where $R_{0}$ stands for the radius of the bent potential interface. We assume that the homogeneous electric potential of strength $V$ is applied on the right side of this interface, while it is 0 on the left-hand side. This barrier is shown schematically in Fig. 2 together with the band structure of graphene.

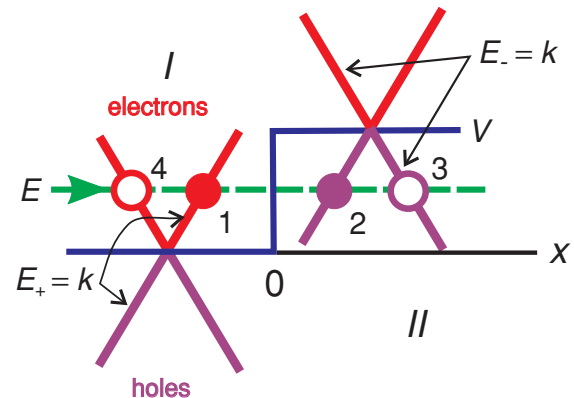

FIG. 2. (Color online) Potential barrier and the band structure of graphene.

We assume that the incident electron beam with energy $E=k_{i}>0$,

$$
\Psi_{i}(\mathbf{r})=\frac{A_{i} e^{i k_{i} x}}{\sqrt{x-w_{i}}}\left(\begin{array}{l}
1 \\
1
\end{array}\right) \exp \left\{\frac{i k_{i} y^{2}}{2\left(x-w_{i}\right)}\right\},
$$

is coming from the left side in region $I$. It corresponds to point 1 , indicated by the filled red circle in Fig. 2. So, in the case of positive $R_{0}$ the interface, Eq. (24), corresponds to a convex lens.

We assume that the energy of the incident electron is lower than the potential height $(E<V)$. Due to conservation of energy and chirality, the electron in the barrier corresponds to point 2 (filled violet circle) in the energy spectrum shown in Fig. 2. Points 3 and 4, shown by open circles, do not contribute in the paraxial approximation due to the above-mentioned conservation of chirality.

So, the wave function on the right side of barrier II reads

$$
\Psi_{t}(\mathbf{r})=\frac{A_{t} e^{i k_{t} x}}{\sqrt{x-w_{t}}}\left(\begin{array}{l}
1 \\
1
\end{array}\right) \exp \left\{\frac{i k_{t} y^{2}}{2\left(x-w_{t}\right)}\right\},
$$

where

$$
k_{t}=E-V<0 .
$$

The incident, Eq. (25), and transmitted, Eq. (26), beams have to satisfy the boundary condition at the interface, Eq. (24), which reads

$$
\begin{aligned}
& \frac{A_{i} e^{i k_{i} y^{2} / 2 R_{0}}}{\sqrt{y^{2} / 2 R_{0}-w_{i}}} \exp \left\{\frac{i k_{i} y^{2}}{2\left(y^{2} / 2 R_{0}-w_{i}\right)}\right\} \\
& =\frac{A_{t} e^{i k_{t} y^{2} / 2 R_{0}}}{\sqrt{y^{2} / 2 R_{0}-w_{t}}} \exp \left\{\frac{i k_{t} y^{2}}{2\left(y^{2} / 2 R_{0}-w_{t}\right)}\right\} .
\end{aligned}
$$

Taking into account the weak divergence of the Gaussian beam and assuming a large radius for the bent interface $\left(R_{0} \gg \lambda\right)$, Eq. (28) can be simplified by neglecting the $y^{2} / 2 R_{0}$ terms in comparison with the $w_{i, t}$ terms in the denominators. This replaces Eq. (28) with the following equation:

$$
\begin{aligned}
& \frac{A_{i}}{\sqrt{-w_{i}}} \exp \left\{i k_{i} y^{2}\left[1 / 2 R_{0}-1 / 2 w_{i}\right]\right\} \\
& =\frac{A_{t}}{\sqrt{-w_{t}}} \exp \left\{i k_{t} y^{2}\left[1 / 2 R_{0}-1 / 2 w_{t}\right]\right\} .
\end{aligned}
$$




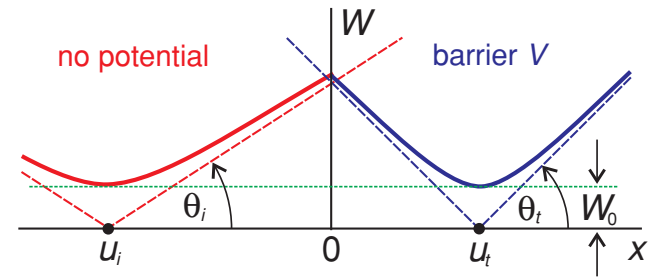

FIG. 3. (Color online) Beam penetration into the flat potential step at $x=0$ for $E<V$.

As this equation has to hold for any $y$ value at the interface, the following condition should be satisfied:

$$
k_{i}\left(\frac{1}{R_{0}}-\frac{1}{w_{i}}\right)=k_{t}\left(\frac{1}{R_{0}}-\frac{1}{w_{t}}\right) .
$$

Note that this is the main equation for lenses and mirrors in optics.

In the case of a flat interface (i.e., $R_{0}=\infty$ ), Eq. (30) reduces to the more simple one,

$$
k_{i} / w_{i}=k_{t} / w_{t}
$$

or

$$
w_{t}=-\kappa w_{i}, \quad \kappa=\frac{V-E}{E}>0 .
$$

Consequently, the positions of the waist of the incident and transmitted beams ( $u_{i}$ and $u_{t}$, respectively) have different signs as illustrated in Fig. 3, where the radii of corresponding beams are shown by solid red and blue curves.

It is remarkable that the flat interface does not influence the radius of the waist. Indeed, according to Eqs. (8) and (31) we have

$$
W_{0 t}=\sqrt{\frac{2 v_{t}}{k_{t}}}=\sqrt{\frac{2 v_{i}}{k_{i}}}=W_{0 i},
$$

while the focusing properties of this interface, namely, the divergence of the beam according to Eq. (9), can be enlarged as shown by

$$
\theta_{0 t}=\frac{2}{k_{t} W_{0 t}}=\frac{k_{i}}{k_{t}} \theta_{0 i}=-\frac{1}{\kappa} \theta_{0 i}
$$

when the energy of the incident electron approaches the top of the barrier. Note that when the electron energy becomes higher than the potential height, the interface loses its focusing possibility because in this case $\kappa<0$, and both beam waists are on the same side of the interface. Now the beam diverges after the penetration into the barrier as illustrated in Fig. 4.

One can change the waist of the beam in the barrier by using a bent interface. In this case, assuming that the waists of both beams are not close to the interface (i.e., $\left|u_{i}\right| \gg v_{i}$ and $\left|u_{t}\right| \gg v_{t}$ ), we can rewrite Eq. (7) as

$$
\frac{1}{w_{i, t}} \approx \frac{1}{u_{i, t}}-\frac{i v_{i, t}}{u_{i, t}^{2}},
$$

and inserting it into Eq. (30) and separating its real and imaginary parts, we obtain two equations,

$$
k_{i}\left(\frac{1}{R_{0}}-\frac{1}{u_{i}}\right)=k_{t}\left(\frac{1}{R_{0}}-\frac{1}{u_{t}}\right),
$$

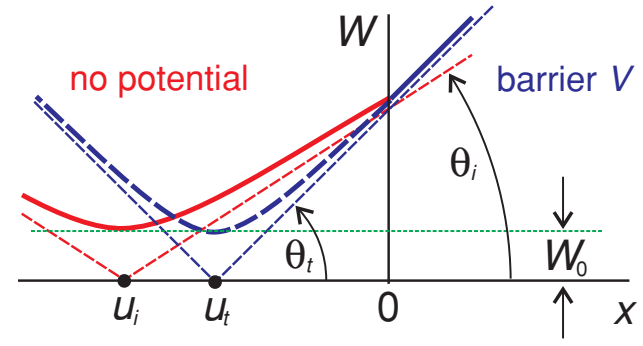

FIG. 4. (Color online) Diverging beam when penetrating the flat potential step at $x=0$ with $E>V$.

$$
\frac{k_{i} v_{i}}{u_{i}^{2}}=\frac{k_{t} v_{t}}{u_{t}^{2}},
$$

which characterize the transition of the Gaussian beam through the bent interface.

Now using Eqs. (8) and (36b), the waist ratio becomes

$$
\frac{W_{0 t}}{W_{0 i}}=\sqrt{\frac{k_{i} v_{t}}{k_{t} v_{i}}}=\frac{k_{i} u_{t}}{k_{t} u_{i}} .
$$

Using Eq. (36a) we can express the ratio $u_{i} / u_{t}$ as

$$
\frac{u_{i}}{u_{t}}=\frac{k_{i}}{k_{t}}\left[1-\left(1-\frac{k_{t}}{k_{i}}\right) \frac{u_{i}}{R_{0}}\right],
$$

and we obtain the following equation for the ratio of the beam waists:

$$
\frac{W_{0 t}}{W_{0 i}}=\left(1-\frac{V u_{i}}{E R_{0}}\right)^{-1} .
$$

We see that in the case of a diverging beam coming from the left side $\left(u_{i}<0\right)$, the convex $\left(R_{0}>0\right)$ interface compress the beam waist similarly to the action of a convex lens in optics, while a concave interface $\left(R_{0}<0\right)$, in contrast, widens it without affecting its focusing ability.

\section{REFLECTION OF AN OBLIQUE BEAM}

Now we consider a standard optics problem: reflection and transmission of a beam obliquely incident on the interface of two different media. The layout for the case of graphene is shown in Fig. 5. In the right half-plane (yellow) there is a barrier - the homogeneous electric potential of strength $V$-while in the left half-plane the potential is taken to be 0 . The vector $\mathbf{k}_{i}$ indicates the direction of the incident beam characterized by the incident angle $\theta_{i}$. The simplest way to construct the wave function that corresponds to that incident

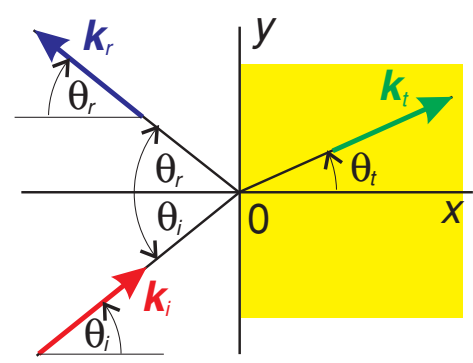

FIG. 5. (Color online) Reflection and transmission at an interface. 
beam is to use the coordinate system $\left(x_{i}, y_{i}\right)$ rotated by the angle $\theta_{i}$, namely,

$$
\begin{gathered}
x_{i}=x \cos \theta_{i}+y \sin \theta_{i}, \\
y_{i}=-x \sin \theta_{i}+y \cos \theta_{i},
\end{gathered}
$$

in which the vector $\mathbf{k}_{i}$ is located along the $x_{i}$ axis. Thus, taking into account that the spinor has to be rotated as well and denoting $E=\left|\mathbf{k}_{i}\right|$, we rewrite Eq. (25) as follows:

$$
\Psi_{i}(\mathbf{r})=\frac{A_{i} e^{i E x_{i}}}{\sqrt{x_{i}-w_{i}}}\left(\begin{array}{c}
1 \\
e^{i \theta_{i}}
\end{array}\right) \exp \left\{\frac{i E y_{i}^{2}}{2\left(x_{i}-w_{i}\right)}\right\} .
$$

In an analogous way, denoting the reflection angle by the symbol $\theta_{r}$ and rotating coordinates by the angle $\pi-\theta_{r}$ in Eq. (25), we obtain the wave function of the reflected beam:

$$
\Psi_{r}(\mathbf{r})=\frac{A_{r} e^{i E x_{r}}}{\sqrt{x_{r}-w_{r}}}\left(\begin{array}{c}
1 \\
-e^{-i \theta_{r}}
\end{array}\right) \exp \left\{\frac{i E y_{r}^{2}}{2\left(x_{r}-w_{r}\right)}\right\} .
$$

The wave function of the transmitted beam is similar to Eq. (41), where the index $i$ has to be replaced by $t$, and the energy $E$ by the electron energy in the barrier $E-V$.

Now satisfying the boundary condition at the interface $(x=0)$, we obtain the equation

$$
\begin{aligned}
& e^{i E y \sin \theta_{i}}\left(\begin{array}{c}
1 \\
e^{i \theta_{i}}
\end{array}\right) \frac{A_{i} \Phi_{i}(E)}{\sqrt{y \sin \theta_{i}-w_{i}}} \\
& +e^{i E y \sin \theta_{r}}\left(\begin{array}{c}
1 \\
-e^{-i \theta_{r}}
\end{array}\right) \frac{A_{r} \Phi_{r}(E)}{\sqrt{y \sin \theta_{r}-w_{r}}} \\
& =e^{i(E-V) y \sin \theta_{t}}\left(\begin{array}{c}
1 \\
e^{i \theta_{t}}
\end{array}\right) \frac{A_{t} \Phi_{t}(E-V)}{\sqrt{y \sin \theta_{t}-w_{t}}},
\end{aligned}
$$

where the following short notation is used:

$$
\Phi_{\alpha}(E)=\exp \left\{\frac{i E y^{2} \cos ^{2} \theta_{\alpha}}{2\left(y \sin \theta_{\alpha}-w_{\alpha}\right)}\right\} .
$$

Equation (43) has to be satisfied for any $y$ value at the interface. This implies that the arguments of the exponents that include the coordinate $y$ have to be equal. So, applying this procedure to the first exponent in all three terms of Eq. (43), we obtain the following equations:

$$
E \sin \theta_{i}=E \sin \theta_{r}=(E-V) \sin \theta_{t} .
$$

This is nothing other than Snell's law:

$$
\theta_{r}=\theta_{i}, \quad \sin \theta_{t}=-\frac{1}{\kappa} \sin \theta_{i} .
$$

If $E<V(\kappa>0)$, we have a negative angle of refraction $\left(\theta_{t}<0\right)$ as in the case of a metamaterial.

To equate the arguments of the second exponent in each of the three terms of Eq. (43) as given by Eq. (44) is hardly possible due to the nontrivial $y$ dependence of the numerator of this expression and of the denominator as well. Therefore, we have to invoke some approximations. For this purpose we draw our attention to definition (6) and Eq. (7), from which it follows that the term $y \sin \theta_{\alpha}$, which actually does not exceed the beam radius, competes with the term $u_{\alpha}$, which indicates the distance from the interface to the beam's waist. Consequently, if the waist is not close to the interface, namely, if the above distance $u_{\alpha}$ is larger than the radius of the beam characterized by the value $v_{\alpha}$, the term $y \sin \theta_{\alpha}$ can be neglected compared with the shift $w_{\alpha}$. Making use of this approximation and equating the arguments of the $\Phi_{\alpha}(E)$-type exponents in all three terms of Eq. (43), we obtain the following equations:

$$
\begin{gathered}
\frac{E \cos ^{2} \theta_{i}}{w_{i}}=\frac{E \cos ^{2} \theta_{r}}{w_{r}}=\frac{(E-V) \cos ^{2} \theta_{t}}{w_{t}} \\
\left(\begin{array}{c}
1 \\
e^{i \theta_{i}}
\end{array}\right) \frac{A_{i}}{\sqrt{-w_{i}}}+\left(\begin{array}{c}
1 \\
-e^{-i \theta_{r}}
\end{array}\right) \frac{A_{r}}{\sqrt{-w_{r}}}=\left(\begin{array}{c}
1 \\
e^{i \theta_{t}}
\end{array}\right) \frac{A_{t}}{\sqrt{-w_{t}}} .
\end{gathered}
$$

These equations completely solve the beam reflection problem. Equation (47a) enables us to define $w_{r}$ and $w_{t}$, namely, the basic characteristics of reflected and transmitted beams:

$$
w_{r}=w_{i}=-\frac{w_{t}}{\kappa} \frac{\cos ^{2} \theta_{i}}{\cos ^{2} \theta_{t}} .
$$

The first equality indicates that the reflected beam has the same characteristics as the incident one; actually the reflected beam is a continuation of the incident one with the same divergence rate. The second equality enables us to estimate the waist of the transmitted beam. It reads

$$
\frac{W_{0 t}^{2}}{W_{0 i}^{2}}=-\frac{1}{\kappa} \frac{v_{t}}{v_{i}}=\frac{\cos ^{2} \theta_{t}}{\cos ^{2} \theta_{i}},
$$

and consequently,

$$
\frac{W_{0 t}}{W_{0 i}}=\frac{\cos \theta_{t}}{\cos \theta_{i}}
$$

Thus the waist of the transmitted beam can be increased (or decreased) compared with the incident beam, depending on the angle of incidence.

And finally, Eq. (47b) enables us to evaluate the intensities of the transmitted and reflected beams. This equation is actually the same equation for homogeneous beams described by the corresponding exponents (see, e.g., similar calculations in Ref. 26) with a single exception, namely, the additional factors $w_{\alpha}$, which actually take into account the change of the beam waist during reflection or transmission.

\section{BEAM COLLIMATION IN A SUPERLATTICE}

Using Eq. (31) that describes the transformation of the main beam parameters when passing through the flat $p-n$ interface it becomes now easy to trace the transmission of the electron beam through the superlattice that is composed as a periodic structure of the above interfaces. Such a superlattice is schematically shown in Fig. 6. We assume that this superlattice

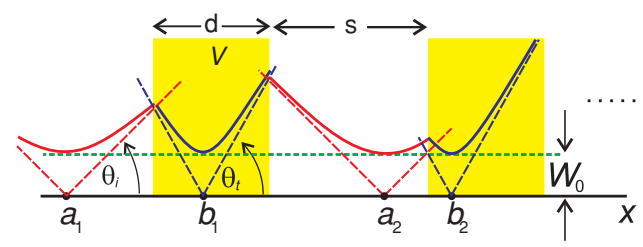

FIG. 6. (Color online) Superlattice and an electron beam propagating through it. 
consists of alternating $n$ regions of length $s$ (shown in white) with zero potential and $p$ regions (or barriers) of length $d$ (shown in yellow) with electric potential $V$. The radius of the electron beam propagating perpendicular to these superlattice layers is shown by solid red and blue curves in Fig. 6. The corresponding dashed lines indicate the divergences of various beam parts. The green dashed horizontal line shows the position of the beam waist, which, according to Eq. (33), maintains its value in the case of the considered flat interfaces.

As the waist value is conserved during electron transmission through the interface indicated by the number $n$, the main variables that characterize this transmission are $a_{n}$ and $b_{n}$, which are the distances of incoming and outgoing beam waists from that interface (see Fig. 6). According to Eq. (32) they are related by

$$
b_{n}=-a_{n} \kappa \quad \text { and } \quad a_{n}=-b_{n} / \kappa .
$$

The single important thing that must be taken into account is that these relations have to be used in the coordinate system attached to the considered interface. Thus, we can write the following set of equations connecting the waist positions in various superlattice layers:

$$
\begin{aligned}
b_{n} & =-\kappa a_{n}, \\
a_{n+1}-s & =-\frac{1}{\kappa}\left(b_{n}-d\right),
\end{aligned}
$$

or

$$
a_{n+1}=a_{n}+\left(\frac{d}{\kappa}-s\right) .
$$

The solution of this simple equation reads

$$
a_{n}=a_{1}+\left(\frac{d}{\kappa}-s\right)(n-1) .
$$

It is remarkable that Eq. (53) and its solution, Eq. (54), hold even in the case where the waist positions are outside the layers that contact the interface under consideration. Thus, it follows from Eq. (54) that, in general, the distance of the beam waist from the interface increases when $n \rightarrow \infty$, and consequently, the electron beam diverges. The single exception is the case where the electron energy satisfies the following condition:

$$
d=\kappa s \quad \text { or } \quad E=\frac{s}{s+d} V .
$$

This is actually the condition of electron beam collimation by means of a superlattice composed of alternating $p-n$ and $n-p$ interfaces.

It is remarkable that the above condition could be formulated within the framework of a quasiclassical approach and actually is not related to band parameters. Previously the collimation of electrons was related to their spectrum. It was argued that the presence of flat regions in the electron energy-momentum spectrum favors collimation ${ }^{16-19,27}$ of beams propagating in the direction perpendicular to those flat regions. The disadvantage of the latter technique was the very small superlattice period (of the order of nanometers) and rather large barrier heights (of the order of tenths of electron volts) that were required to observe collimation (see, e.g., Ref. 18). Note that the quasiclassical Gaussian beams presented are free of this disadvantage.

\section{CONCLUSIONS}

We have rewritten the Gaussian beam expressions used in optics and adjusted them for the description of Dirac electrons. The application of Gaussian beams was illustrated by considering electron beam transmission through simple nonhomogeneous structures.

It was shown that although a $p$ - $n$ interface in graphene exhibits a negative refraction for an electron beam, as in an optical metamaterial, it does not influence the electron beam waist, which is the essential feature of any lens focusing of optical rays. A contraction of the beam waist, however, is possible if one uses a bent $p-n$ interface, which actually resembles a real lens.

Looking for possible electron beam collimation, we applied our results to electron propagation in a superlattice and obtained a general condition under which such a collimation can be realized. We showed that collimation is possible in the case of larger lattice constants and smaller barrier heights than previously predicted.

\section{ACKNOWLEDGMENTS}

This research was supported by the Flemish Science Foundation (Grant No. FWO-Vl), by the Belgian Science policy (IAP), and (in part) by the Lithuanian Science Council under project No. MIP-79/2010.

\footnotetext{
*Corresponding author: amatulis@takas.lt

†mrmphys@gmail.com

${ }^{\ddagger}$ francois.peeters@ua.ac.be

${ }^{1}$ T. Chakraborty, Quantum Dots (Elsevier, Amsterdam, 1999).

${ }^{2}$ K. S. Novoselov, A. K. Geim, S. V. Morozov, D. Jing, M. I. Katsnelson, I. V. Grigorieva, S. V. Dubonos, and A. A. Firsov, Nature (London) 438, 197 (2005).

${ }^{3}$ Y. Zang, Y. W. Tan, H. L. Stormer, and P. Kim, Nature (London) 438, 201 (2005).

${ }^{4}$ J. Moore, Nature (London) 464, 194 (2010).
}

${ }^{5}$ P. R. Wallace, Phys. Rev. 71, 622 (1947).

${ }^{6}$ V. V. Cheianov, V. Fal'ko, and B. L. Al'tshuler, Science 315, 1252 (2006).

${ }^{7}$ A. G. Moghaddam and M. Zareyan, Phys. Rev. Lett. 105, 146803 (2010).

${ }^{8}$ F. Hassler, A. R. Akhmerov, and C. W. J. Beenakker, Phys. Rev. B 82, 125423 (2010).

${ }^{9}$ J. C. Meyer, C. O. Girit, M. F. Crommie, and A. Zettl, Appl. Phys. Lett. 92, 123110 (2008).

${ }^{10}$ S. Marchini, S. Günther, and J. Wintterlin, Phys. Rev. B 76, 075429 (2007). 
${ }^{11}$ A. L. Vazquez de Parga, F. Calleja, B. Borca, M. C. G. Passeggi Jr., J. J. Hinarejos, F. Guinea, and R. Miranda, Phys. Rev. Lett. 100, 056807 (2008).

${ }^{12}$ Y. Pan, N. Jiang, J. Sun, D. Shi, S. Du, F. Liu, and H.-J. Gao, Adv. Mater. 21,2777 (2009).

${ }^{13}$ H. van Houten, B. J. van Wees, J. E. Mooij, C. W. J. Beenakker, J. G. Williamson, and C. T. Foxon, Europhys. Lett. 5, 721 (1988).

${ }^{14}$ J. Spector, H. L. Stormer, K. W. Baldwin, L. N. Pfeiffer, and K. W. West, Appl. Phys. Lett. 56, 1290 (1990).

${ }^{15}$ U. Sivan, M. Heiblum, C. P. Umbach, and H. Shtrikman, Phys. Rev. B 41, R7937 (1990).

${ }^{16}$ C.-H. Park, Y.-W. Son, L. Yang, M. L. Cohen, and S. G. Louie, Nano Lett. 8, 2920 (2008).

${ }^{17}$ M. Barbier, P. Vasilopoulos, and F. M. Peeters, Phys. Rev. B 81, 075438 (2010).

${ }^{18}$ C.-H. Park, L. Yang, Y.-W. Son, M. L. Cohen, and S. G. Louie, Nat. Phys. 4, 213 (2008).
${ }^{19}$ M. Barbier, P. Vasilopoulos, and F. M. PeetersPhys. Rev. B 80, 205415 (2009).

${ }^{20}$ M. Ramezani Masir, P. Vasilopoulos, and F. M. Peeters, New J. Phys. 11, 095009 (2009).

${ }^{21}$ C.-H. Park, F. Giustino, M. L. Cohen, and S. G. Louie, Nano Lett. 9, 1731 (2009).

${ }^{22}$ A. Yacoby, M. Heiblum, V. Umansky, H. Shtrikman, and D. Mahalu, Phys. Rev. Lett. 73, 3149 (1994).

${ }^{23}$ G. M. Maksimova, V. Ya. Demikhovskii, and E. V. Frolova, Phys. Rev. B 78, 235321 (2008).

${ }^{24}$ V. Ya. Demikhovskii, G. M. Maksimova, A. A. Perov, and E. V. Frolova, Phys. Rev. A 82, 052115 (2010).

${ }^{25}$ B. E. A. Saleh and M. C. Teich, Fundamentals of Photonics (John Wiley \& Sons, New York, 1991), Chap. 3.

${ }^{26}$ M. I. Katsnelson, K. S. Novoselov, and A. K. Geim, Nat. Phys. 2, 620 (2006).

${ }^{27}$ K. Staliunas and R. Herrero, Phys. Rev. E 73, 016601 (2006). 